\begin{tabular}{|c|c|}
\hline Title & Observations of the Velocity Curves of NGC-1068 \\
\hline Author(s) & Kaneko, Noboru; Satoh, Tetsuya; Toy ama, Kiyotaka; Sasaki, Minoru; Nishimura, Masaki; Y amamoto, Masafumi \\
\hline Citation & A stronomical Journal, 103(2), 422-428 \\
\hline Issue Date & $1992-02$ \\
\hline Doc URL & http:/hdl. handle.net/2115/6063 \\
\hline Rights & Copyright $\odot 1992$ A merican Institute of Physics \\
\hline Type & article \\
\hline File Information & A J103_2.pdf \\
\hline
\end{tabular}

Instructions for use 


\title{
OBSERVATIONS OF THE VELOCITY CURVES OF NGC 1068
}

\author{
NOBORU KANEKO \\ Department of Physics, Faculty of Science, Hokkaido University, Sapporo 060, Japan
}

TETSUYA SATOH

Kutchan Agricultural High School, Kutchancho, Abutagun, Hokkaido 044, Japan

KIYOTAKA TOYAMA

Hokkaido Information University, Nishi-Nopporo 59-2, Ebetsu 069, Japan

Minoru SASAKI

Shimonoseki City University, Shimonoseki 751, Japan

MASAKI Nishimura

Soengakuen Research Institute of Computer Science, Chuoku N4 W12, Sapporo 060, Japan

MASAFUMI YAMAMOTO

Fuji Photo Film Co., Ltd., Development Center, Kaiseimachi, Kanagawa 258, Japan

Received 15 May 1991; revised 24 September 1991

\begin{abstract}
For 132 spectra of NGC 1068 taken at 24 position angles of every $15^{\circ}$ from an isophotal major axis $\left(\mathrm{P} . \mathrm{A} .=52^{\circ}\right.$ ) of the inner arm region, we measured $\mathrm{H} \alpha, \mathrm{H} \beta,[\mathrm{N} \mathrm{II}] \lambda 6583$, and [ $\left.\mathrm{O}_{\mathrm{III}}\right] \lambda \lambda 4959,5007$ to present the velocity curves in tabular form. Comparisons are made with previous observations to show that our measurements are consistent with the recent observations, but inconsistent with Walker's measurements. Combined with CO velocity data, the velocity field was analyzed to reveal large-scale noncircular motions to occur within the galaxy.
\end{abstract}

\section{INTRODUCTION}

A precise knowledge of the large-scale velocity field of NGC 1068 is very important to understand the starburst activity taking place within the disk of the galaxy and its linkage with the nuclear activity.

Kinematic observations of NGC 1068 have been published by many authors, but most of them were concerned with spectra in limited numbers of position angles (Burbidge et al. 1959; Bertola 1965; Galletta \& Recillas-Cruz 1982; Alloin et al. 1983; Meaburn \& Pedlar 1986) or presented no results of measurements in tabular form (Atherton et al. 1985; Baldwin et al. 1987; Bergeron et al. 1989; Bland \& Cecil 1989; Cecil et al. 1990). As far as we know, tabularform rotation curve data of NGC 1068 have been given only by Walker (1968). He has measured the velocity curves at 12 position angles of every $30^{\circ}$, and his data have been often used in kinematic studies of NGC 1068 (Scoville et al. 1983; Telesco \& Decher 1988). However, Baldwin et al. (1987) have recently pointed out that their measurements are inconsistent with his results.

Using spectra collected in our observation program to study the large-scale velocity field of NGC 1068, we have already reported the discovery of a highly ionized nebulosity (Nishimura et al. 1984). In this paper we present new measurements of the velocity curves of NGC 1068 in tabular form, and analyze them together with $\mathrm{CO}$ velocity data.

\section{OBSERVATIONS AND REDUCTIONS}

We have used the Cassegrain image-tube spectrograph attached to the $1.88 \mathrm{~m}$ reflector at the Okayama Astrophysical Observatory. 60 blue $(4000-5800 \AA)$ and 72 red $(5700-$
$7400 \AA$ ) spectra were obtained at 24 position angles of every $15^{\circ}$ from an isophotal major axis $\left(\mathrm{P} . \mathrm{A} .=52^{\circ}\right)$ of the inner arm region (Nishimura et al. 1984). Eight to twelve spectra were gathered at each position angle. Exposure time ranges from 3 to $60 \mathrm{~min}$. Spectra were exposed with the nucleus at the center or the edge of the slit $\left(70^{\prime \prime}\right.$ in length and 0.9 or 1.8 in width). Most spectra were obtained using a grating of 600 grooves $\mathrm{mm}^{-1}$ which gives the dispersion of $109 \AA \mathrm{mm}^{-1}$ at $\mathrm{H} \alpha$. A grating of 1800 grooves $\mathrm{mm}^{-1}$ was also used for some spectra. Examples of the blue and red spectra taken along the major axis have been shown in Fig. 1 of Nishimura et al. (1984). A list of the spectrograms will be given elsewhere.

The spectra were measured at intervals of $50 \mu \mathrm{m}(1 " 83)$ on the plate along $\mathrm{H} \alpha, \mathrm{H} \beta,[\mathrm{N} \mathrm{II}] \lambda 6583$, and [O III] $\lambda \lambda 4959,5007$ with the two coordinate measuring machine. The center of the nucleus was taken to be the center of the strip of the continuum of the spectra. We could not measure the lines at the central three positions $(R=0$ " and \pm 1.83$)$ even on the plates of shortest exposure, due to their broad and complex features superposed on the background continuum. The final error in the measurements of a single point has been estimated to be better than $30 \mathrm{~km} \mathrm{~s}^{-1}$. Velocities referred to in this paper are heliocentric.

\section{RESULTS}

We present all measured velocities in Figs. 1(a)-1(c), where 5585 points are plotted and the mean velocities at each point are shown connected by solid lines. No different symbols are used for different spectral lines. This is partly because of the avoidance of the confusion in the figures. Baldwin et al. (1987) pointed out in their Fig. 14 that the 

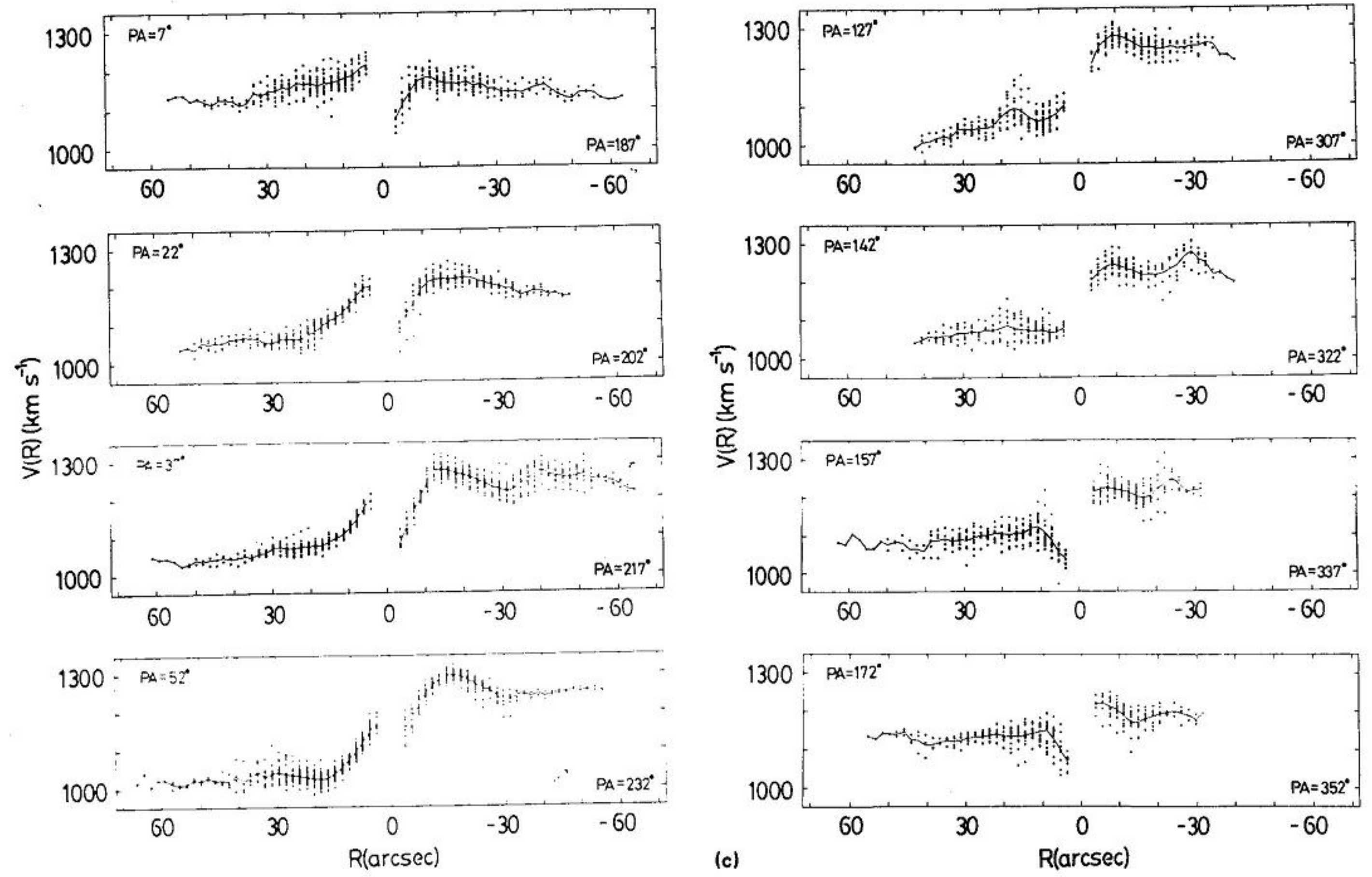

(a)
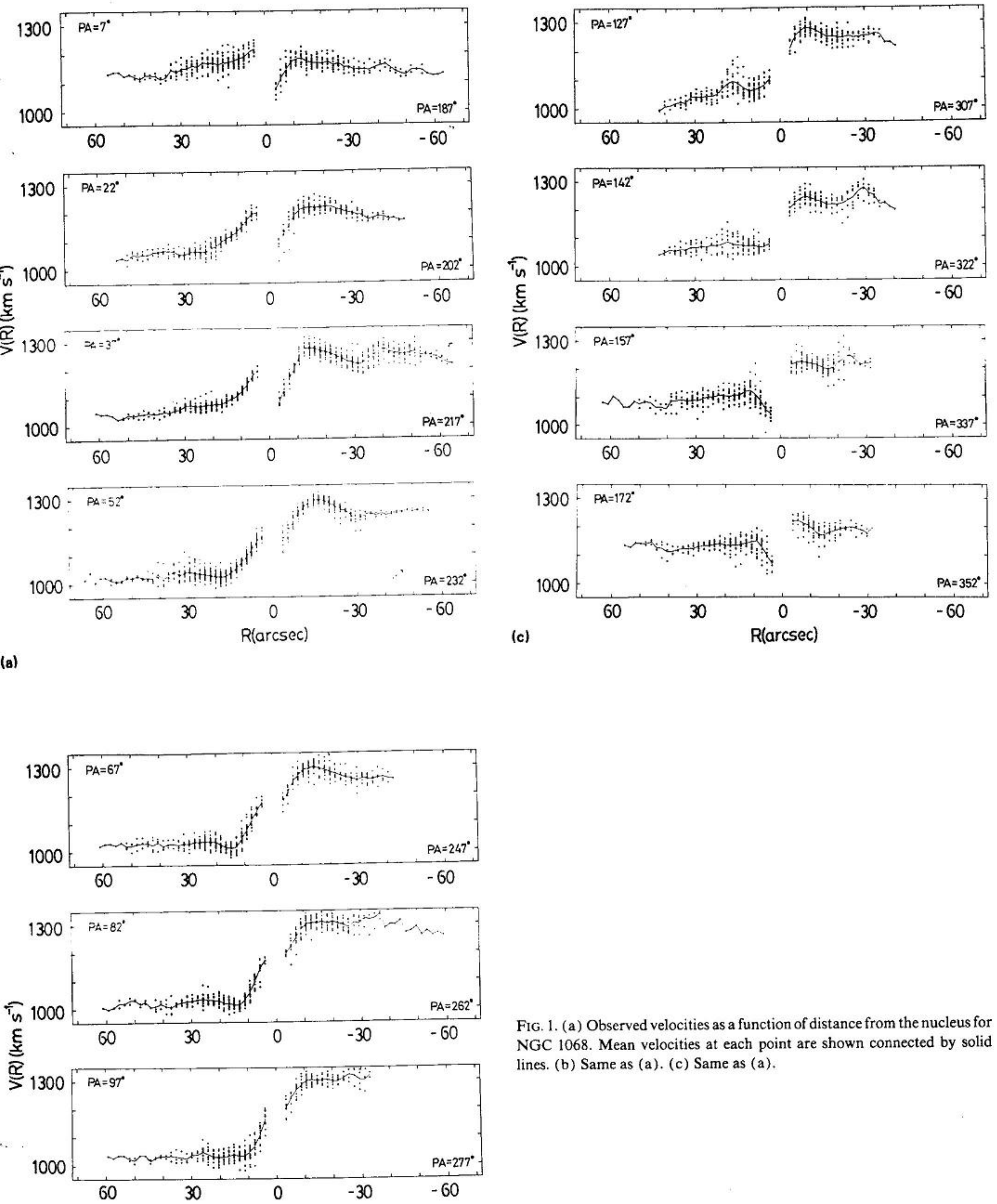

FIG. 1. (a) Observed velocities as a function of distance from the nucleus for NGC 1068. Mean velocities at each point are shown connected by solid lines. (b) Same as (a). (c) Same as (a).

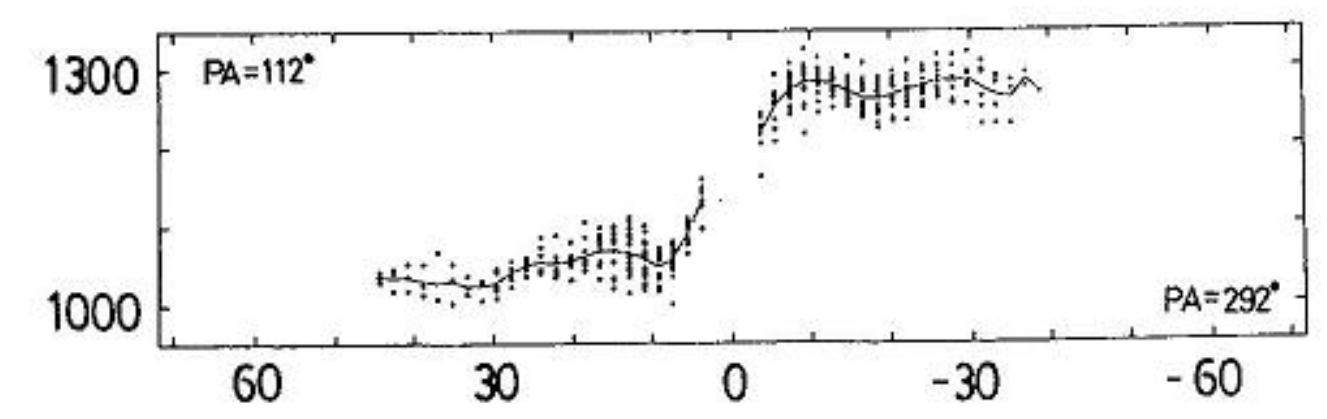

(b)

$\mathrm{R}(\operatorname{arcsec})$ 
velocities of [O III] $\lambda 5007$ are slightly redshifted $(\leqslant 40$ $\mathrm{km} \mathrm{s}^{-}$, relative to $\mathrm{H} \beta$ at all locations. We examined at each position angle the velocity differences for [ $\mathrm{N}$ II] i. $6583-\mathrm{H} \alpha \cdot \mathrm{H} \alpha-\mathrm{H} \beta$. and $\left[\mathrm{O} \mathrm{HI}^{\prime}\right.$; $; 500^{-}-\mathrm{H} \beta$. and took an average of them. Resuitant averages are 0.2 and 10 $\mathrm{km} \mathrm{s}^{-}$for [ $\left.\mathrm{X} \mathrm{HI}\right] ; 658 \mathrm{i}-\mathrm{H} \alpha . \mathrm{H} \alpha-\mathrm{H} \beta$. and [O III] $\lambda 5007-\mathrm{H} \beta$, respectively. We also confirmed that the velocity differences for [ $\mathrm{O}$ III] $i 5007-\mathrm{H} \beta$ are positive at most position angles, which is consistent with the trend pointed out by Baldwin et al. (1987).

However, these differences are small compared with the uncertainty in our measurements, and we are interested in a global velocity structure. In this paper we are concerned with the mean velocities of various spectral lines measured at each point. Table 1 lists the mean velocities, each $1^{\prime \prime} 83$ along 24 position angles of every $15^{\circ}$ from P.A. $=52^{\circ}$, where the figure in parentheses indicates the number of points averaged at each position.

Figure 2 is the soveicity coniour map draw n irom our results, where $U$-band isophotes of the inner arm region are superposed for comparison.

Comparison with optical images (Sandage 1961; Arp 1966; Ichikawa et al. 1987) reveals that the shape of the region where emission lines were measured bears a resemblance to the spiral structures, implying that our measurements almost cover the line emitting disk. The extensions of velocity curves up to $R \sim 60^{\prime \prime}$ in the northeast quadrant correspond to a broad feature of the east spiral arm. The short extensions in the northwest quadrant are attributed to a taillike feature northwardly stretching out from knot No. 4 of Schild et al. (1985).

Large irregularities are found in our velocity curves, which are generally asymmetric with respect to the center. Peaks at $R \sim 10^{\prime \prime}-15^{\prime \prime}$ and dips at $R \sim 20^{\prime \prime}-30^{\prime \prime}$ on the west side coincide in radius with an unusually bright west inner

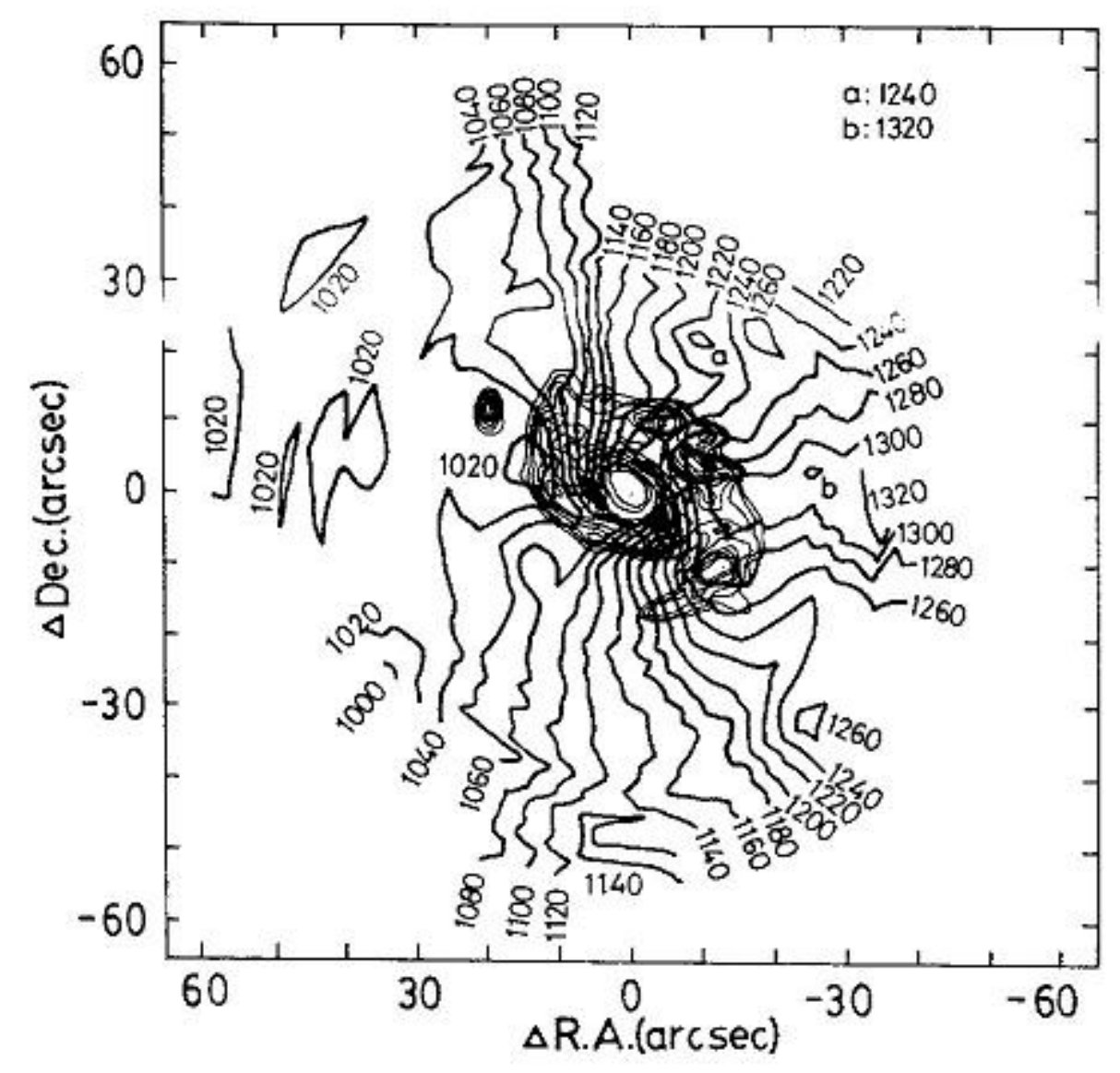

FIG. 2. Isovelocity contour map superposed on the $U$-band isophotes of the inner arm region in NGC 1068. Contours are spaced by $20 \mathrm{~km} \mathrm{~s}^{-1}$. Isophotes were drawn using the plate NS 1005 taken at the Newtonian focus of the Okayama $1.88 \mathrm{~m}$ reflector in November 1968 . spiral (Keel \& Weedman 1978; Snijders et al. 1986) and a heavily obscured dust lane (Sandage 1961), respectively. A peak at $R \sim 40^{\prime \prime}$ on the velocity curve of P.A. $=217^{\circ}$ agrees with knot No. $4\left(R=40^{\prime \prime}\right.$, P.A. $\left.=218^{\circ}\right)$ of Schild et al. (1985). Dips at $R-10^{\prime \prime}-15^{\prime \prime}$ on the velocity curves of P.A. $=52^{\circ}-112^{\circ}$ are correspondent with a sharp dust feature bordering a southern boundary of the inner arm region. Redshifted velocity irregularities shown in Fig. 2 of Bergeron et al. (1989) are seen in the northeast area located between $R=20^{\prime \prime}-40^{\prime \prime}$ and P.A. $=37^{\circ}-82^{\circ}$, which correspond to emission patches lying on the east spiral. It seems likely that the morphologies of both highly ionized clouds and dusty clouds, possibly with which molecular clouds are associated, are closely related with velocity departures from the circular motion.

There is also an indication that the velocity structure within $\sim 10^{\prime \prime}$ from the center is markedly different from that outside $\sim 10^{\prime \prime}$ radius. In the southern portion of the $\sim 10^{\prime \prime}$ central the observed velocities sharply decrease toward the center, suggesting that the kinematic major axis rotates by about $90^{\circ}$ from P.A. $\simeq 90^{\circ}$ as discussed in the next section.

\section{DISCUSSION}

\subsection{Comparison with Previous Observations}

Comparison with earlier observations show that the velocities measured by Burbidge et al. (1959) are higher by $50-100 \mathrm{~km} \mathrm{~s}^{-1}$ while Bertola's (1965) measurements are lower by about $50 \mathrm{~km} \mathrm{~s}^{-1}$ than ours. Velocities measured by Galletta \& Recillas-Cruz (1982) show a large scatter, and are smaller by about $100 \mathrm{~km} \mathrm{~s}^{-1}$ on the northeast than ours.

Comparison with Walker's (1968) observations is very important because his data has been often used in kinematic studies of NGC 1068 (Scoville et al. 1983; Telesco \& Decher 1988). The result is shown in Fig. 3. Except for P.A. $=100^{\circ}$, agreement is good on the east side, but not good on the west side, where his measurements are lower by about 50-100 $\mathrm{km} \mathrm{s}^{-1}$ than ours.

Atherton et al. (1985) have presented the Fabry-Perot observations. Analyzing long-slit spectra, Baldwin et al. (1987) have mentioned that these long-slit and interferometer observations are consistent with $20 \mathrm{~km} \mathrm{~s}^{-1}$ accuracy. We compared our measurements with the above two results, by drawing velocity curves from their maps. Except for a conflicting region pointed out by Baldwin et al. (1987), our results are found to be in good agreement with the two data. In that region our measurements are consistent with those of Baldwin et al. (1987). Bergeron et al. (1989) have presented an off-center velocity curve along P.A. $=37^{\circ}$. Excellent agreement is found at the points where our slits cross their off-center slit. Although no quantative comparison is done, our isovelocity contour map (Fig. 2) appears globally consistent with the Fabry-Perot map of Bland \& Cecil (1989).

\subsection{Comparison with CO Observations}

Kaneko et al. (1989) have presented the CO velocity data obtained with the Nobeyama Radio Observatory (NRO) 45 $\mathrm{m}$ telescope of which the beam size is $17^{\prime \prime}$ (FWHM), and argued that the velocity structure of $\mathrm{CO}$ emission cannot be explained only in terms of a circular motion. Using their Table 2, we plot in Fig. 4 the $\mathrm{CO}$ peak and mean velocities at position angles of every $45^{\circ}$. Comparison with optical velocity curves reveals a global agreement on the east. A tendency 


\begin{tabular}{|c|c|c|c|c|c|c|c|c|c|c|c|c|c|c|c|c|c|c|c|c|c|c|c|c|}
\hline \multirow{2}{*}{$R\left({ }^{\prime}\right)$} & \multicolumn{24}{|c|}{ Position Angle } \\
\hline & $r$ & 27 & $37^{\circ}$ & 52 & $670^{\circ}$ & 824 & 97 & 112 & $127^{\circ}$ & $162^{\circ}$ & $150^{\circ}$ & 172 & $187^{\circ}$ & 202 & 217 & $23 \pi$ & 267 & $26 ?$ & $2 \pi$ & $x$ & זדצ & 㜔 & $: 37$ & Z5 \\
\hline
\end{tabular}

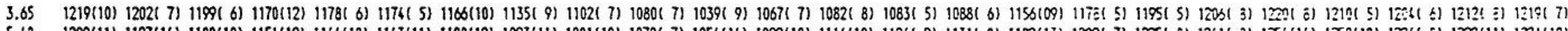

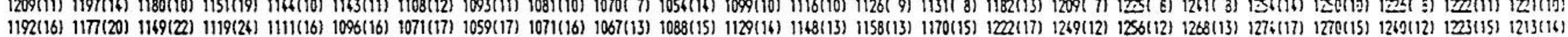

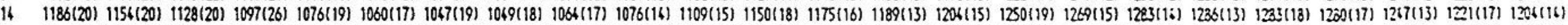

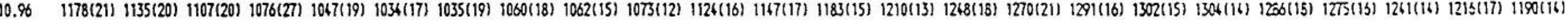

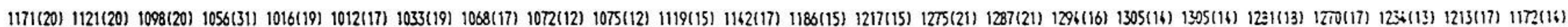

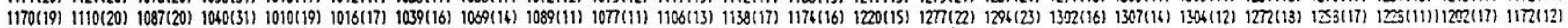

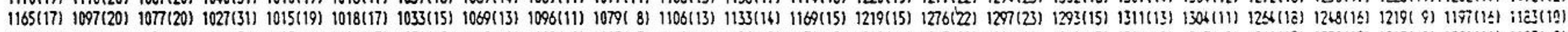

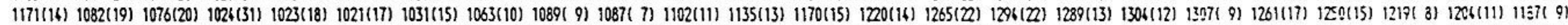

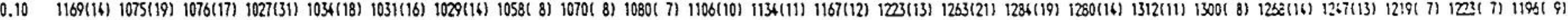

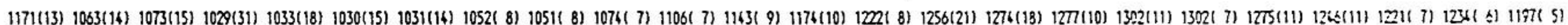

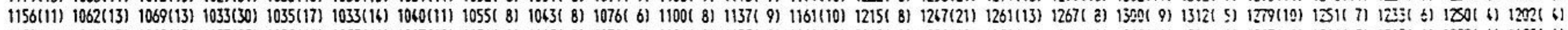

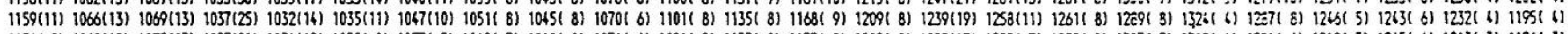

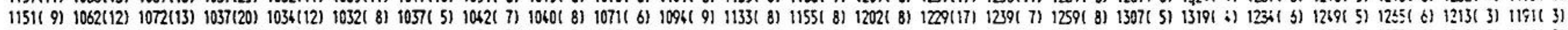

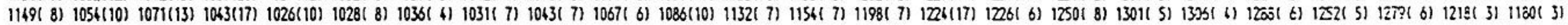

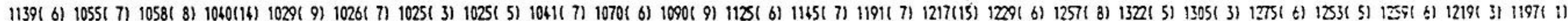

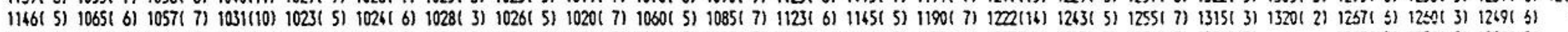

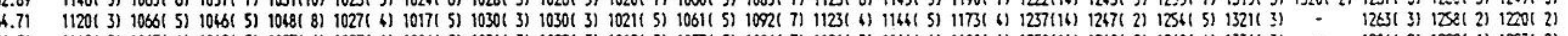

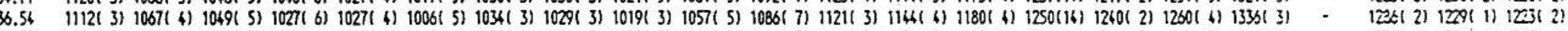

38.37 1126(3) $1064($ 4) $1044(3) 1022$ (4) $1019($ 4) $1012($ 6) $1032($ 2) $1032(3) 1009(3) 1061(2) 1086(7) 1114(3) 1154(3) 1185($ 6) $1260(16) 1246(2) 1262(3) 1254(1)$

$1127(3) 1062(4) 1041(3) 1035(4) 1033(5) 1018(3) 1033(2) 1037(3) 1007(3) 1052(2) 1057(2) 1112(3) 1157(3) 1184($ 4) $1275(10) 1239(2) 1257(2) 1298(1)$

$(5.50$ (1123( 2) $1051($ 4) $1061(3) 1022($ () $1028($ 4) $1027(2) 1036(2)$

$1124(1) 1037(2) 1040(2) 1020($ \&) $1021(4) 1036(2) 1018(2)$ 1138( 1) $1043(2) 1030(2) 1027(3) 1020($ 4) $1031(2) 1023(2)$ 1139 (I) 1037 (1) 1025 (1) 1012 (3) 10321 i) 1020 (2) 1035 (1)

$56.811132(1)-1038(1) 1010(3) 1024(1) 102(2) 1035(1)$

56.64

- - $10441111015(3) 1030(1) 1007($ i) 10281 11

$58.66 \quad-\quad-\quad 1063($ I) $1025(1) 1029(1) 1000($ i) $1035($ i)

$60.29 \quad-\quad-\quad 1050$ ( 1) 1028 ( 1) $1020(1) 1007(1)-$

62.12

63.95

- 1006 (1)

- $\quad 1006(1)$

$\therefore \quad-\quad-\quad 1042(1)$

Hotes - The figure in parentheses indicates the number of points averaged at each position. 


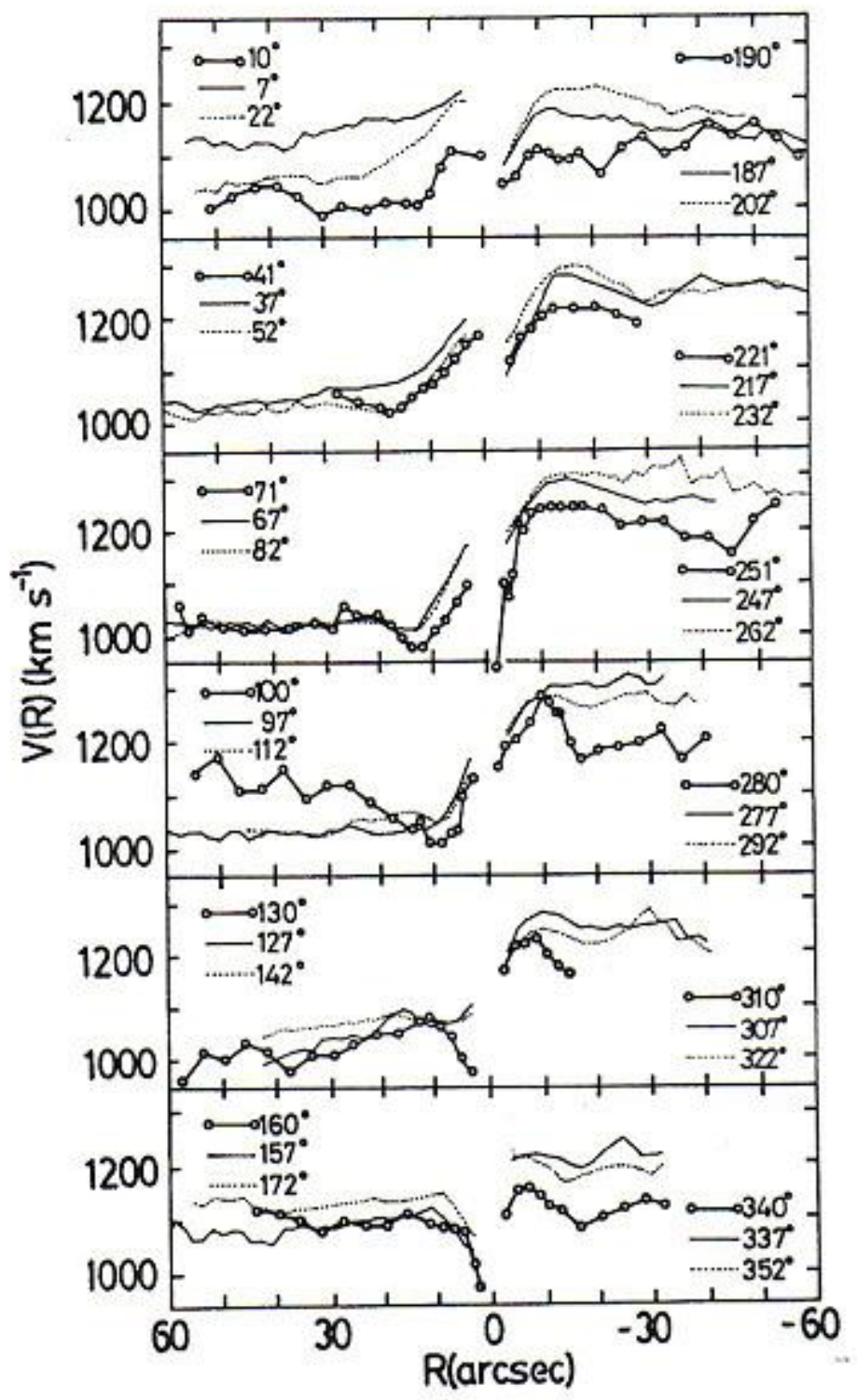

FIG. 3. Comparison with Walker's measurements. Open circles indicate the velocities measured by Walker (1968). The velocities presently being reported are shown by thin solid and dotted lines. is found on the westside that $\mathrm{CO}$ velocities are smaller by about $50-100 \mathrm{~km} \mathrm{~s}^{-1}$ than the optical ones.

In the above comparison the effects of beam smearing are important, but their accurate evaluation is difficult because they depend both on the intensity and velocity structures of $\mathrm{CO}$ emission within the beam. Comparing with a new $\mathrm{CO}$

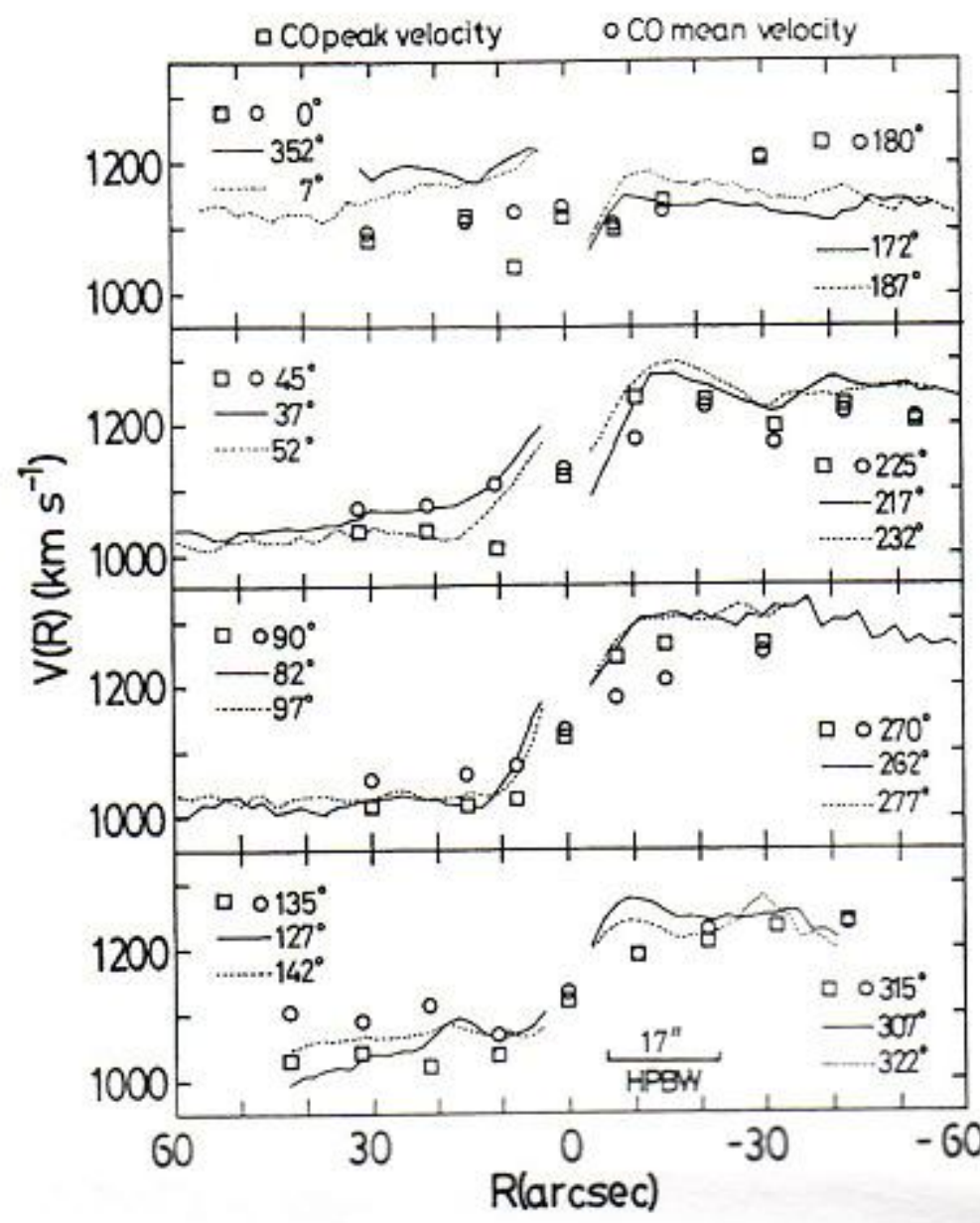

FIG. 4. Comparison with the $\mathrm{CO}$ peak and mean velocities. Solid and dotted lines indicate our velocity curves. The $\mathrm{CO}$ peak and mean velocities, which are, respectively, denoted by open squares and circles, are plotted at position angles of every $45^{\circ}$, by using Table 2 of Kaneko et al. (1989). The HPBW (17") of the Nobeyama Radio Observatory $45 \mathrm{~m}$ telescope with which their observations were made is denoted by the horizontal bar. observations with the Nobeyama Millimeter Array (NMA) (Kaneko et al. 1991), we estimate that the position accuracy of the CO velocities plotted in Fig. 4 is of order $\sim 5^{\prime \prime}-8^{\prime \prime}$.

We show in Fig. 5 superposition of the velocity field (Fig. 2) with the CO map obtained with the NMA (Kaneko et al. 1991). The striking feature of the figure is that distortions of
TABLE 2. Published systemic velocities of NGC 1068.

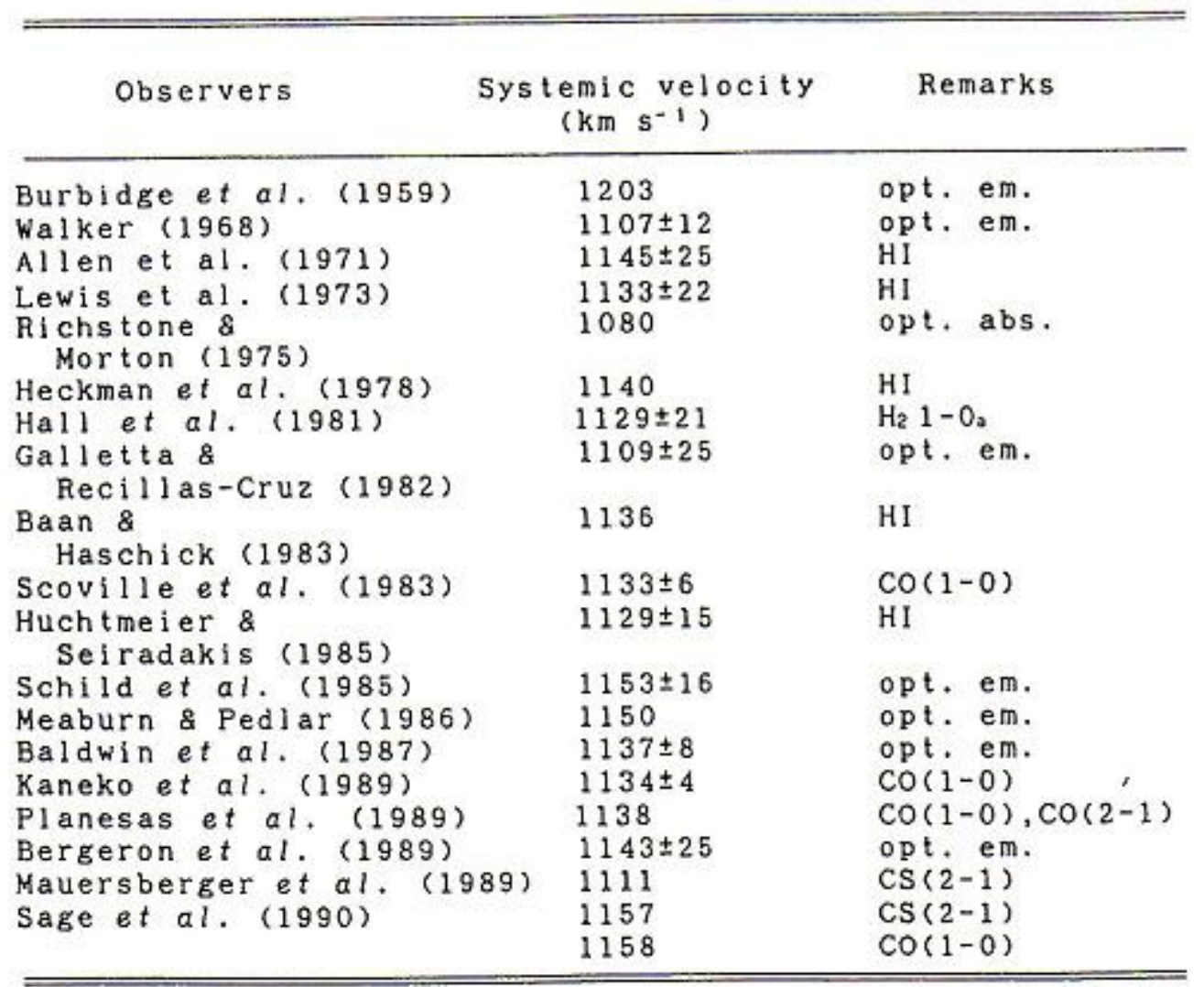

a f $\mid u x$ mean of $S(0)$ and $S(1)$

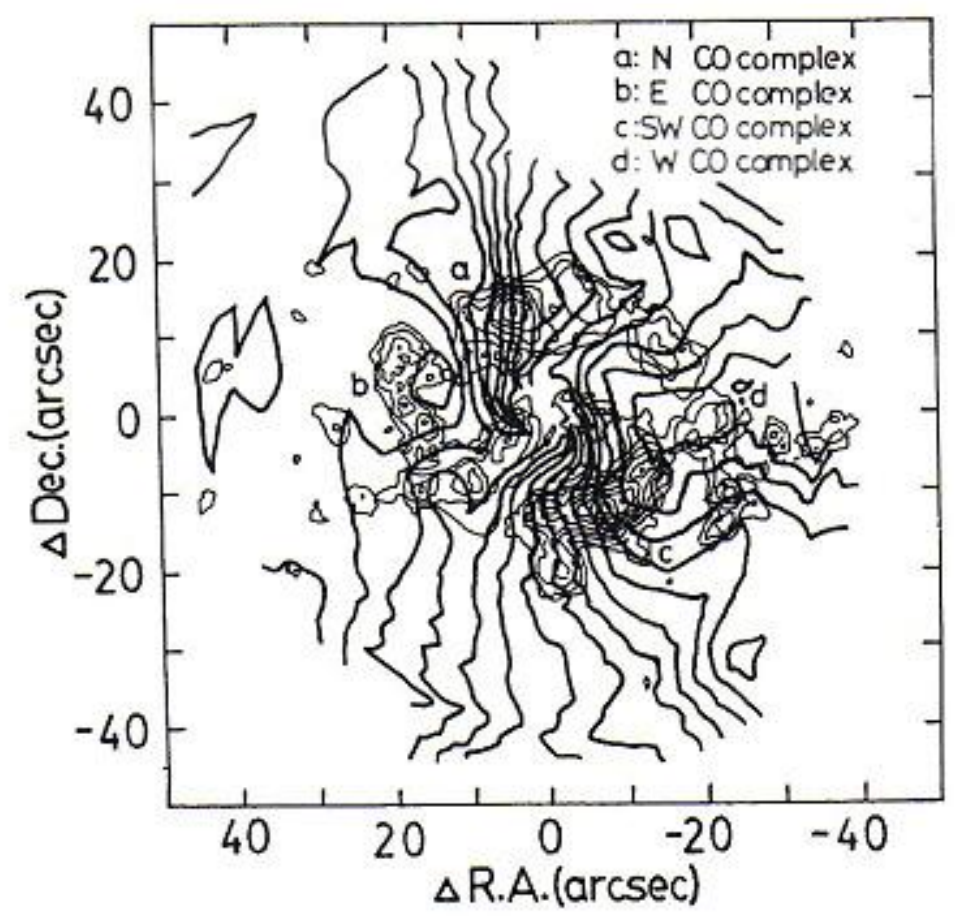

FIG. 5. Superposition of the velocity field (Fig. 2) on the $\mathrm{CO}$ interferometer map obtained with the Nobeyama Millimeter Array (Kaneko et al. 1991). This interferometer map is very different from that of Planesas et al. (1991). 
the isovelocity contours trace well the $\mathrm{CO}$ molecular cloud complexes. This suggests a close relation between the starforming activity and velocity departures from the circular motion.

\subsection{Major Axis and Galaxy Inclination}

We plot in Fig. 6 the optical and CO velocities as a function of position angle, where a fitted cosine curve, $V$ $\left(\mathrm{km} \mathrm{s}^{-1}\right)$ the velocity corresponding to a systemic velocity, $A\left(\mathrm{~km} \mathrm{~s}^{-1}\right)$ the velocity amplitude, and $\phi\left(^{\circ}\right)$ the major axis position angle are given for each velocity range. Outside the $\sim 10^{\prime \prime}$ central, both optical and CO velocities clearly show that the kinematic major axis lies in the east-west direction, consistently with the recent results (Baldwin et al. 1987; Bergeron et al. 1989). This position angle of the kinematic major axis is consistent with the morphology of the envelope (Burbidge et al. 1959; Hodge 1968; Kaneko et al. 1989), but inconsistent with the morphology of the main spiral disk (Sandage 1961; Arp 1966; Ichikawa et al. 1987). The rotation of the kinematic major axis within $\sim 10$ " radius is also clearly seen in Fig. 6. One of the kinematic peculiarities of NGC 1068 is that the kinematic major axis does not coincide with the isophotal one which changes progressively with the distance from the center (Pomea \& Rieke 1990).

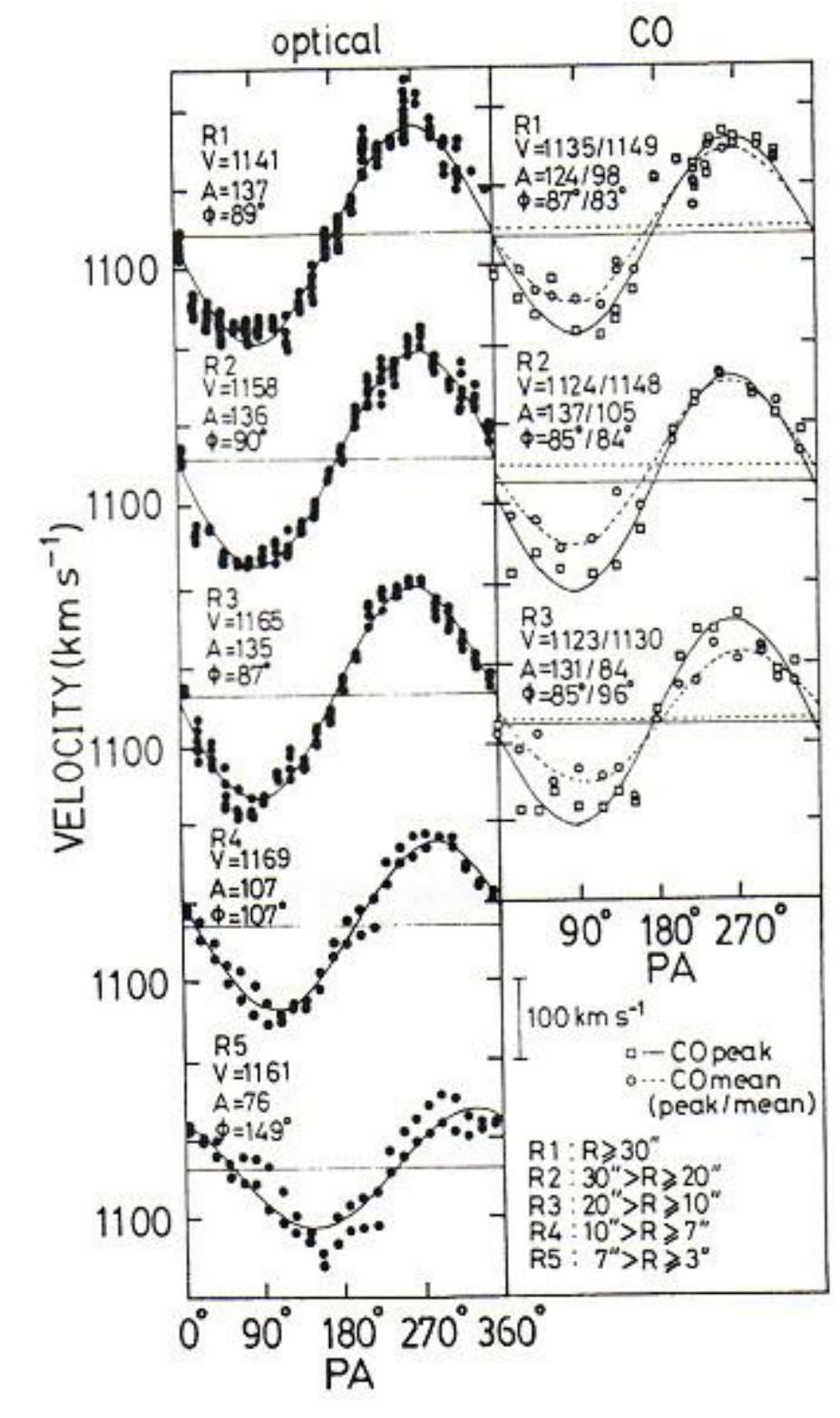

FIG. 6. Optical and $\mathrm{CO}$ velocities as a function of position angle. Filled circles indicate our optical velocities. Open squares and circles denote the $\mathrm{CO}$ peak and mean velocities (Kaneko et al. 1989), respectively. The velocities were fitted with a cosine curve, and the velocity $V$ $\left(\mathrm{km} \mathrm{s}^{-1}\right)$ corresponding to a systemic velocity, the velocity amplitude $A\left(\mathrm{~km} \mathrm{~s}^{-1}\right)$, and the major axis position angle $\phi\left({ }^{\circ}\right)$ are given for each velocity range.

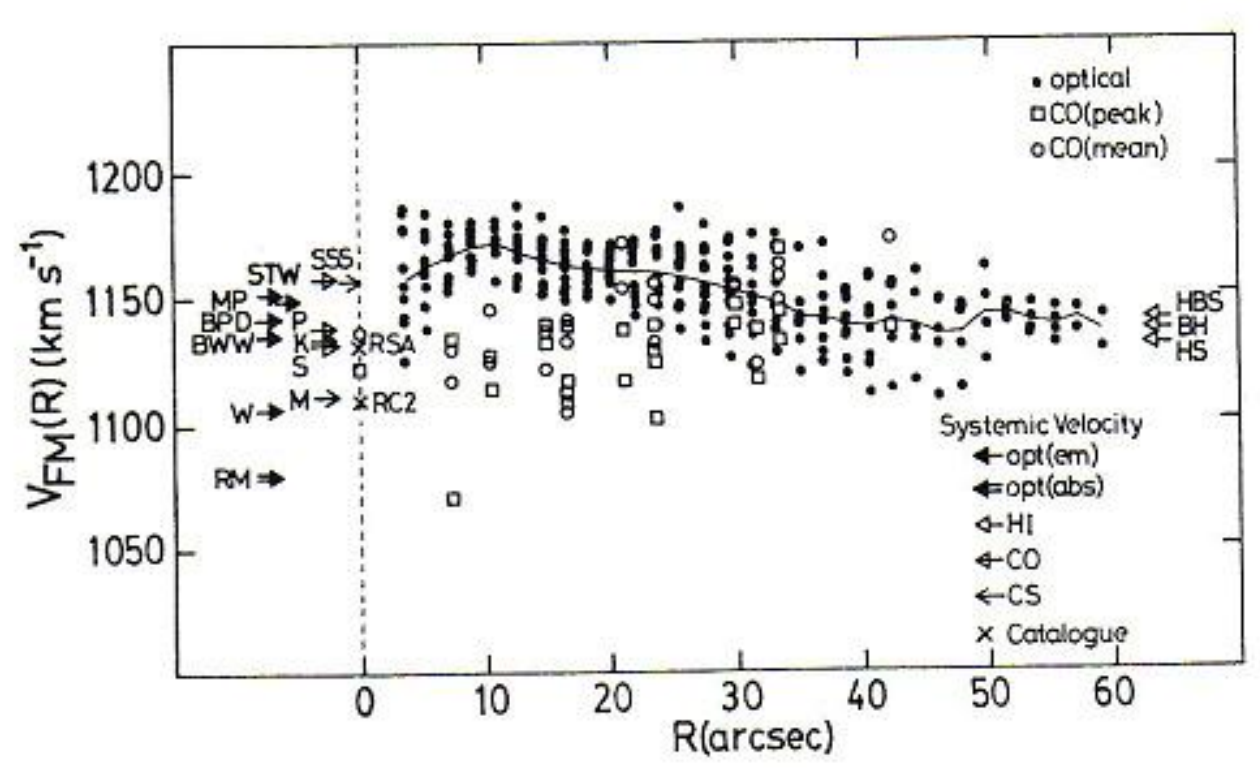

FIG. 7. The folded and mean velocities as a function of distance from the center. The folded and mean (FM) velocities are described in the text. Filled circles indicate the optical FM velocities, and the mean values at each point are shown connected by solid lines. Open squares and circles denote the FM velocities calculated from the $\mathrm{CO}$ peak and mean velocities (Kaneko et al. 1989), respectively. Arrows are the published systemic velocities denoted by BH (Baan \& Haschick 1983), BPD (Bergeron et al. 1989), BWW (Baldwin et al. 1987), HBS (Heckman et al. 1978), HS (Huchtmeier \& Seiradakis 1985), K (Kaneko et al. 1989), M (Mauersberger et al. 1989), MP (Meaburn \& Pedlar 1986), P (Planesas et al. 1989), RM (Richstone \& Morton 1975), S (Scoville et al. 1983), SSS (Sage et al. 1990), STW (Schild et al. 1985), and W (Walker 1968). Two crosses denote the catalogued values: $1109 \mathrm{~km} \mathrm{~s}^{-1}$ (RC2) and $1131 \mathrm{~km} \mathrm{~s}^{-1}$ (RSA).

Assuming a rotation law, we tried to determine the galaxy inclination $i$, together with the other parameters. The result gives $i \sim 0^{\circ}$. This is because the velocity field is unusually flat on the east. If we restrict ourselves to the west half side, we derive $i \sim 20^{\circ}$, in agreement with the results of Baldwin et al. (1987) and Bergeron et al. (1989). However, these small inclinations yield a deprojected amplitude larger than 400 $500 \mathrm{~km} \mathrm{~s}^{-1}$, too large for a typical Sb galaxy (Rubin et al. $1982,1985)$. Alternatively, adoption of $i \sim 35^{\circ}-40^{\circ}$ consistent with the morphology of the envelope (Hodge 1968; Kaneko et al. 1989) deforms a shape of the spiral disk into an unusually prolonged bar. Thus we have no good inclination consistent both with the kinematics and the morphology.

\subsection{Systemic Velocity and Noncircular Motions}

We define a folded and mean velocity (FM) as the mean of two velocities at the positions symmetric with respect to the center (Kaneko \& Satoh 1989). The FM velocities defined in this way should agree with a systemic velocity provided that no noncircular motions are significant. We plot in Fig. 7 the optical and CO FM velocities, where published systemic velocities (Table 2) are marked for comparison.

Optical FM velocities show a peak of about $1175 \mathrm{~km} \mathrm{~s}^{-1}$ at $R \sim 10^{\prime \prime}$, which corresponds to the luminous inner spirals. Extrapolation of them gives about $1145 \mathrm{~km} \mathrm{~s}^{-1}$ at $R=0^{\prime \prime}$, consistently with the systemic velocities determined from optical emission lines (Schild et al. 1985; Meaburn \& Pedlar 1986; Baldwin et al. 1987; Bergeron et al. 1989). Optical FM velocities show an outward decrease, and become flat outside $\sim 30^{\prime \prime}$ radius. As shown in Fig. 6, we have $V=1141$ $\mathrm{km} \mathrm{s}^{-1}$ for $R \geqslant 30^{\prime \prime}$. We note that this is in good agreement with the H I velocities (Heckman et al. 1978; Baan \& Haschick 1983; Huchtmeier \& Seiradakis 1985). On the other hand, CO FM velocities that are inside $\sim 20$ " radius are 
considerably lower, and at $R \sim 30^{\prime \prime}$ they reach the same level as the optical ones.

If the $\mathrm{H}$ I velocity is a good indicator of the systemic velocity, we may adopt a velocity of $1141 \mathrm{~km} \mathrm{~s}^{-1}$ for the systemic velocity of NGC 1068. Noncircular motions are then considered to be dominant within a radius of $\sim 30^{\prime \prime}$. Alternatively, if the systemic velocity is close to $1080 \mathrm{~km} \mathrm{~s}^{-1}$ determined from stellar absorption lines (Richstone \& Morton 1975), departure from the circular motion is supposed to occur within the whole galaxy.

\section{CONCLUDING REMARKS}

We have presented the velocity curves, each 1 ". 83 along 24 position angles of every $15^{\circ}$ from the isophotal major axis $\left(\right.$ P.A. $\left.=52^{\circ}\right)$ of the inner arm region. We expect that our tabular-form data may be useful in kinematic studies of NGC 1068.
Comparison with previous observations shows that our measurements are consistent with the recent observations, but inconsistent with those of Walker (1968). Careful treatments might be needed in case his velocity data and kinematic parameters are adopted.

Analysis of the velocity field reveals that large-scale noncircular motions occur within the galaxy. This is essential in discussing a bar-driven density wave scenario (Scoville et al. 1988: Telesco \& Decher 1988; Thronson et al. 1989), a merger hypothesis (Myers \& Scoville 1987; Kaneko \& Satoh 1989), and other triggering mechanisms. However, quantative properties of the noncircular motions still remain uncertain, because we have no knowledge of the velocity field of the background stellar system.

We are grateful to the staff of the Okayama Astrophysical Observatory for help with the observations.

\section{REFERENCES}

Allen, R. J., Darchy, B. F., \& Lauqué, R. 1971, A\&A, 10, 198

Alloin, D., Pelat, D., Boksenberg, A., \& Sargent, W. L. W. 1983, ApJ, 275, 493

Arp, H. 1966, Atlas of Peculiar Galaxies (California Institute of Technology, Pasadena)

Atherton, P. D., Reay, N. K., \& Taylor, K. 1985, MNRAS, 216, 17P

Baan, W. A., \& Haschick, A. D. 1983, AJ, 88, 1088

Baldwin, J., Wilson, A. E., \& Whittle, M. 1987, ApJ, 319, 84

Bergeron, J., Petitjean, P., \& Durret, F. 1989, A\&A, 213, 61

Bertola, F. 1965, A\&A, 28, 574

Bland, J., \& Cecil, G. N. 1989, in Active Galactic Nuclei, IAU Symposium No. 134, edited by D. E. Osterbrock and J. S. Miller (Kluwer, Dordrecht), p. 343

Burbidge, E. M., Burbidge, G. R., \& Prendergast, K. H. 1959, ApJ, 130, 26

Cecil, G., Bland, J., \& Tully, R. B. 1990, ApJ, 355, 70

de Vaucouleurs, G., de Vaucouleurs, A., \& Corwin, Jr., H. G. 1976, Second Reference Catalogue of Bright Galaxies (University of Texas Press, Austin) ( $\mathrm{RC2}$ )

Galletta, G., \& Recillas-Cruz, R. B. 1982, A\&A, 112, 361

Hall, D. N. B., Kleinmann, S. G., Scoville, N. Z., \& Ridgway, S. T. 1981, ApJ, 248, 898

Heckman, T. M., Balick, B., \& Sullivan III, W. T. 1978, ApJ, 224, 745

Hodge, P. W. 1968, AJ, 73, 846

Huchtmeier, W. K., \& Seiradakis, J. H. 1985, A\&A, 143, 216

Ichikawa, S., Okamura, S., Kaneko, N., Nishimura, M., \& Toyama, K. 1987, PASJ, 39, 411

Kaneko, N., Morita, K., Fukui, Y., Sugitani, K., Iwata, T., Nakai, N., Kaifu, N., \& Liszt, H. S. 1989, ApJ, 337, 691

Kaneko, N., and Satoh, T. 1989, in Active Galactic Nuclei, IAU Symposium No. 134, edited by D. E. Osterbrock and M. J. Miller (Kluwer, Dordrecht), p. 357
Kaneko, N., Morita, K., Fukui, Y., Takahashi, N., Sugitani, K., Nakai, N., \& Morita, K.-I. 1991, PASJ (submitted)

Keel, W. C., \& Weedman, D. W. 1978, AJ, 83, 1

Lewis, B. M., \& Davies, R. D. 1973, MNRAS, 165, 213

Mauersberger, R., Henkel, C., Wilson, T. L., \& Harju, J. 1989, A\&A, 226, L5

Meaburn, J., \& Pedlar, A. 1986, A\&A, 159, 336

Myers, S. T., \& Scoville, N. Z. 1987, ApJ, 312, L39

Nishimura, M., Kaneko, N., \& Toyama, K. 1984, A\&A, 130, 46

Planesas, P., Gomez-Gonzales, J., \& Martin-Pintado, J. 1989, A\&A, 216, 1

Planesas, P., Scoville, N., \& Myers, S. T. 1991, ApJ, 369, 364

Pompea, S. M., \& Rieke, G. H. 1990, ApJ, 356, 416

Richstone, D. O., \& Morton, D. C. 1975, ApJ, 201, 289

Rubin, V. C., Ford, Jr., W. K., \& Thonnard, N. 1982, ApJ, 261, 439

Rubin, V. C., Burstein, D., Ford, Jr., K. F., \& Thonnard, N. 1985, ApJ, 289, 81

Sage, L. J., Shore, S. N., \& Solomon, P. M. 1990, ApJ, 351, 422

Sandage, A. 1961, The Hubble Atlas of Galaxies (Carnegie Institution of Washington, DC)

Sandage, A., \& Tammann, G. A. 1981, Revised Shapley-Ames Catalog of Bright Galaxies (Carnegie Institution of Washington, Washington, DC) (RSA)

Schild, R., Tresh-Fienberg, R., \& Huchra, J. 1985, AJ, 90, 411

Scoville, N. Z., Young, J. S., \& Lucy, L. B. 1983, ApJ, 270, 433

Scoville, N. Z., Matthews, K., Carico, D. P., \& Sanders, D. B., 1988, ApJL, 327, L61

Snijders, M. A. J., Netzer, H., \& Boksenberg, A. 1986, MNRAS, 222, 549

Telesco, C. M., \& Decher, R. 1988, ApJ, 334, 573

Thronson, H. A., et al. 1989, ApJ, 343, 158

Walker, M. F. 1968, ApJ, 151, 71 